\title{
Oxidative Balance Score and Chronic Kidney Disease
}

\author{
Titilayo O. Ilori Young Sun Ro So Yeon Kong Orlando M. Gutierrez \\ Akinlolu O. Ojo Suzanne E. Judd K.M. Venkat Narayan Michael Goodman \\ Laura Plantinga William McClellan
}

Emory University School of Medicine, Atlanta, Georgia

\section{Key Words}

Oxidative balance $\cdot$ Pro-oxidant $\cdot$ Antioxidant $\cdot$ Chronic kidney disease $\cdot$ End-stage renal disease $\cdot$ Albuminuria

\begin{abstract}
Background: The oxidative balance score (OBS) is a composite estimate of the overall pro- and antioxidant exposure status in an individual. The aim of this study was to determine the association between OBS and renal disease. Methods: Using the Reasons for Geographic and Racial Differences in Stroke cohort study, OBS was calculated by combining 13 a priori-defined pro- and antioxidant factors by using baseline dietary and lifestyle assessment. OBS was divided into quartiles (Q1-Q4) with the lowest quartile, Q1 (predominance of pro-oxidants), as the reference. Multivariable logistic regression and Cox proportional hazards models were used to estimate adjusted ORs for albuminuria defined as urine albu$\mathrm{min} / \mathrm{creatinine}$ ratio $(\mathrm{ACR})>30 \mathrm{mg} / \mathrm{g}$, macroalbuminuria defined as $A C R>300 \mathrm{mg} / \mathrm{g}$ and chronic kidney disease (CKD) defined as estimated glomerular filtration rate $<60 \mathrm{ml} /$ $\mathrm{min} / 1.73 \mathrm{~m}^{2}$ according to the Chronic Kidney Disease Epidemiology Collaboration and hazards ratios for end-stage renal disease (ESRD), respectively. Results: Of the 19,461 participants analyzed, $12.9 \%$ had albuminuria and $10.1 \%$ had CKD at baseline; over a median follow-up of 3.5 years (range 2.144.32 years), $0.46 \%$ developed ESRD. Higher OBS quartiles were associated with lower prevalence of CKD (OR vs. Q1:
\end{abstract}

$\mathrm{Q} 2=0.93$ [95\% Cl 0.80-1.08]; Q3 = 0.90 [95\% Cl 0.77-1.04] and Q4 = 0.79 [95\% Cl 0.67-0.92], p for trend <0.01). The associations between OBS and albuminuria ( $p$ for trend 0.31 ) and incident ESRD ( $p$ for trend 0.56 ) were not significant in the fully adjusted models. Conclusions: These findings suggest that higher OBS is associated with lower prevalence of CKD. Lack of association with ESRD incidence in the multivariable analyses indicates that temporal relation between OBS and renal damage remains unclear.

C) 2015 S. Karger AG, Basel

\section{Introduction}

Oxidative stress has been implicated both in the pathogenesis of chronic kidney disease (CKD) and in cardiovascular complications of CKD such as atherosclerosis [1-4]. Oxidative stress is defined as an imbalance between pro- and antioxidants in favor of pro-oxidants [5]. CKD is associated with increased levels of reactive oxygen species and nitrogen species $[5,6]$. This process is observed in early CKD and may contribute to both progressive decline in the glomerular filtration rate (GFR) and vascular complications [6-8]. Individuals with CKD and compromised nutritional status have more evidence of oxidative stress than the well-nourished ones $[9,10]$.

The balance between pro- and antioxidants is largely determined by endogenous enzymatic mechanisms [11,

\section{KARGER 125\%}

(c) 2015 S. Karger AG, Basel

$0250-8095 / 15 / 0424-0320 \$ 39.50 / 0$
Titilayo O. Ilori, MD

Renal Division, Emory University School of Medicine 1639 Pierce Drive Clifton Road

Atlanta 30322 (Georgia)

E-Mail tilori@emory.edu 
12]. However, exogenous factors such as diet, medications and lifestyle are major environmental sources for both pro- and antioxidants [13]. Ascertaining the individual effects of oxidative balance-related exposures is difficult because they are small, may be correlated and there may be existing biological interaction involving pro- and antioxidant factors [14]. In 2002, Van Hoydonck et al. [15] proposed an oxidative balance score (OBS) as a composite measure of oxidative stress-related exposures. OBS uses information about the dietary micronutrient pro- and antioxidants, antioxidant medication use and pro-oxidative smoking status to summarize pro- and antioxidant exposure status $[15,16]$. Data from population-based studies on pro- and antioxidant exposure status in individuals with CKD are sparse and focus on individual micro and macronutrients $[17,18]$ or dietary patterns [19] and their association with outcomes such as end-stage renal disease (ESRD) and mortality.

The OBS has been shown to correlate inversely with all-cause mortality [15], and with the risk of sporadic colorectal adenoma $[12,20,21]$ and colorectal cancer [22]. However, the associations between OBS and measures of CKD, including estimated GFR (eGFR), albuminuria and incident ESRD, have not been previously studied.

\section{Subjects and Methods}

\section{Study Design}

The Reasons for Geographic and Racial Differences in Stroke (REGARDS) study is a national, population-based prospective study to examine reasons for variation in stroke incidence and mortality in the United States. Details on recruitment and data collection were reported previously [23]. Briefly, between January 2003 and October 2007, more than 30,000 healthy volunteers over the age of 45 years were randomly selected and recruited with planned equal recruitment of men and women and oversampling of African Americans and residents of the 8 southeastern US states because they have higher stroke mortality than the rest of the United States. These states are divided into the 'stroke buckle' (coastal plain regions of Georgia, North and South Carolina) and the 'stroke belt' (the remaining regions of Georgia, North and South Carolina, Mississippi, Alabama, Tennessee, Louisiana and Arkansas) [19]. Trained personnel collected data by using a computer-assisted telephone survey to obtain sociodemographic factors and other information. Subsequently, a health professional visited the participants' homes to collect anthropomorphic variables as well as blood and urine samples. At this appointment, a 1998 Block Food Frequency Questionnaire (98-block FFQ; Nutrition Quest, Berkeley, Calif., USA) was left with the participant to be self-administered and returned to the data coordinating center. Various versions of the block FFQ had been validated in different populations and found to correlate with diet records, most correlations being in the $0.5-0.6$ range [24]. Although compared to diary entry for nutritional intake, FFQ tends to underestimate micronutrient intake while the total energy intake is overestimated but this measurement error is distributed evenly across study sub-populations [25-27].

The REGARDS study was approved by the institutional review boards at all the participating centers and all participants provided informed consent.

Participants were then contacted by telephone every 6 months to capture outcomes of interest such as stroke. ESRD status was assessed by linkage with the United States Renal Data System (USRDS). The USRDS is a registry of ESRD, and it captures over 95\% of incident cases in the United States [28].

\section{Population}

From a total number of 30,239 participants, we excluded 8,686 individuals who did not have data from the block FFQ (28.6\%). Of the remaining 21,553 , we excluded individuals who were found on record linkage to have already been treated for $\operatorname{ESRD}(n=23)$ at the time of baseline interview, those with missing albumin/creatinine ratio (ACR; $n=943)$, then those with missing eGFR $(n=576)$ and those with missing data on any OBS component $(n=550)$. A total of 19,461 participants were included in the final analysis. Excluded individuals differed only by race and were predominantly black (57.6\%).

\section{OBS}

The main exposure (OBS) was calculated by summing up 13 a priori-defined pro- and antioxidant exposure factors (table 1) as described previously $[13,15]$. These factors include intake of polyunsaturated fatty acid (PUFA), iron, selenium, vitamin C, vitamin $\mathrm{E}, \alpha$-carotene, $\beta$-carotene, lutein, lycopene, and cryptoxanthin intake; and use of aspirin, non-steroidal anti-inflammatory drugs (NSAIDs) and alcohol [15]. Smoking was excluded from the original OBS because smoking is a well-known strong risk factor for CKD [29-34]. A higher OBS is reflective of higher antioxidant levels and low pro-oxidant levels and a lower score is reflective of lower antioxidant levels and higher pro-oxidant levels. The dietary data were collected at baseline using the FFQ, which consists of 107 items that assesses dietary intake over the previous year. The FFQ has been described in detail in previous studies [19, 35]. Completed questionnaires were scanned and sent to Nutrition Quest ${ }^{\mathrm{TM}}$ (Berkeley, Calif., USA) for analysis of nutrition content.

The continuous dietary variables reflecting pro-oxidant (unsaturated fat and iron) and antioxidant (vitamin $\mathrm{C}$, lycopene, $\alpha$-carotene, $\beta$-carotene, lutein, $\beta$-cryptoxanthin, vitamin $E$ and selenium) exposures were divided into low, medium and high categories based on each exposure's sex-specific tertiles. For antioxidants, the 1 st through 3 rd tertiles were assigned 0 through 2 points, respectively, whereas the corresponding point assignment for prooxidants was the reverse ( 0 points for the highest tertile and 2 points for the lowest tertile).

A similar scoring approach was used for pro- and antioxidant categorical variables. For antioxidant aspirin and NSAID use, 0 points were assigned to participants with no regular use, 1 point to those with unknown or missing data, and 2 points to those with regular use. For pro-oxidant alcohol consumption, nondrinkers, moderate drinkers (1-7 drinks/week for women and 1-14 drinks/week for men) and heavy drinkers ( $>7$ drinks/week for women and $>14$ drinks/week for men) received 2, 1 and 0 points, respectively (table 1). 
Table 1. OBS components

\begin{tabular}{|c|c|}
\hline 1. Cryptoxanthin & $0=$ low $(1$ st tertile $), 1=$ medium $(2$ nd tertile $), 2=$ high $(3 \mathrm{rd}$ tertile $)$ \\
\hline 3. Aspirin & $0=$ never, $1=$ missing, $2=$ current user \\
\hline 4. Other NSAIDs & $0=$ never, $1=$ missing, $2=$ current user \\
\hline $\begin{array}{l}\text { 5. Alcohol consumption } \\
\text { Female } \\
\text { Male }\end{array}$ & $\begin{array}{l}- \\
0=8+\text { drinks/week, } 1=1-7 \text { drinks/week, } 2=<1 \mathrm{drink} / \text { week } \\
0=15+\text { drinks/week, } 1=1-14 \text { drinks } / \text { week, } 2=<1 \mathrm{drink} / \text { week }\end{array}$ \\
\hline 6. PUFA & $0=$ high $(3 \mathrm{rd}$ tertile $), 1=$ medium $(2$ nd tertile $), 2=$ low $(1$ st tertile $)$ \\
\hline 7. Total (dietary and supplemental) iron & $0=$ high $(3 \mathrm{rd}$ tertile $), 1=$ medium $(2$ nd tertile $), 2=$ low $(1$ st tertile $)$ \\
\hline 8. Total (dietary and supplemental) vitamin $\mathrm{C}$ & $0=$ low $(1$ st tertile), $1=$ medium $(2$ nd tertile $), 2=$ high $(3 \mathrm{rd}$ tertile $)$ \\
\hline 9. Total (dietary and supplemental) $\alpha$-carotene & $0=$ low $(1$ st tertile), $1=$ medium $(2$ nd tertile), $2=$ high $(3 \mathrm{rd}$ tertile $)$ \\
\hline 12. Lutein & $0=$ low $(1$ st tertile $), 1=$ medium $(2$ nd tertile $), 2=$ high $(3 \mathrm{rd}$ tertile $)$ \\
\hline 13. Lycopene & $0=$ low $(1$ st tertile $), 1=$ medium $(2$ nd tertile $), 2=$ high $(3$ rd tertile $)$ \\
\hline
\end{tabular}

Low, medium and high categories correspond to sex-specific 1st, 2nd and 3rd tertiles. NSAID = Non-steroidal anti-inflammatory drug (not including aspirin); PUFA = polyunsaturated fatty acid.

\section{Outcome Measures}

We defined albuminuria as ACR $>30 \mathrm{mg} / \mathrm{g}$ at baseline. Macroalbuminuria was defined as ACR $>300 \mathrm{mg} / \mathrm{g}$. CKD was defined as eGFR $<60 \mathrm{ml} / \mathrm{min} / 1.73 \mathrm{~m}^{2}$ calculated by the Chronic Kidney Disease Epidemiology Collaboration (CKD-EPI) equation. The eGFR at baseline was calculated using the CKD-EPI [36] equation using isotope dilution mass spectrometry-calibrated creatinine. Incident ESRD was determined by linkage of the REGARDS study participants to the USRDS via personal identifiers. ESRD was defined as chronic renal failure requiring permanent renal replacement therapy such as dialysis or transplant [28]. ESRD status was determined through August 31, 2009.

\section{Covariates}

Age, sex, race, smoking, income and educational attainment were self-reported. Region was classified as stroke belt, stroke buckle and other; caloric intake was obtained from the FFQ and analyzed as a continuous variable. We adjusted for systolic blood pressure (SBP), diastolic blood pressure (DBP), baseline eGFR and total cholesterol as continuous variables. Coronary artery disease (CAD) was defined as history of heart disease (self-reported myocardial infarction), coronary artery bypass, angioplasty or stenting OR evidence of myocardial infarction via electrocardiogram. Diabetes was defined as fasting blood glucose $>126 \mathrm{mg} / \mathrm{dl}$, non-fasting blood glucose $>200 \mathrm{mg} / \mathrm{dl}$ or self-reported use of insulin or oral hypoglycemic agents. Statin use was self-reported. The waist circumference was measured in centimeters during the in-home visit. Physical activity was assessed by asking about the number of times participants engaged in intense physical activity enough to work up a sweat (we assessed physical activity as 1-3 times a week and above vs. other).

\section{Statistical Analysis}

The baseline characteristics of the study population were reported for the overall population and across OBS quartiles. Differences in baseline characteristics among the 4 OBS quartiles were tested using analysis of variance for continuous variables and chisquare statistics for categorical variables.

In all analyses, the exposure was OBS (in quartiles and as a continuous variable) and the dependent variables of interest were albuminuria, macroalbuminuria, CKD and ESRD. The first OBS quartile (predominance of pro-oxidants) was used as the reference group. ORs and 95\% CIs were estimated using logistic regression for albuminuria and CKD. Adjusted hazards ratios (HRs) and 95\% CIs were estimated using the Cox proportional hazards model for ESRD. We tested the $\mathrm{PH}$ assumption by examination of log-log survival curves, Schoenfeld residuals and the extended Cox models and found that the $\mathrm{PH}$ assumption was satisfied. The trend tests for OBS (from lower to higher quartiles) in the crude and adjusted models were analyzed by assessing the OBS quartiles as an ordinal variable.

We developed 2 models to adjust for various confounders based on prior literature review. Model 1 adjusted for age, sex, race, region and calories, and model 2 adjusted for variables in model 1 plus body mass index, smoking, waist circumference, physical activity, education, income, SBP, DBP, total cholesterol, CAD, diabetes and statin medications. In studying ESRD, we also adjusted for albuminuria and baseline eGFR in addition. All reported p values are 2-sided with an alpha of 0.05 .

We conducted sensitivity analysis with NSAIDs and aspirin removed from the OBS, and we also investigated the association between the individual components of the OBS and albuminuria and CKD. 
Table 2. Characteristics of participants in the REGARDS cohort by OBS quartiles at baseline (higher quartiles represent higher antioxidant exposure)

\begin{tabular}{|c|c|c|c|c|c|}
\hline Characteristic & All & \multicolumn{4}{|l|}{ OBS quartile } \\
\hline $\mathrm{n}$ & 19,462 & 4,096 & 5,090 & 5,453 & 4,823 \\
\hline Age & $64.8(9.2)$ & $63.5(9.3)$ & $64.4(9.3)$ & $65.2(9.1)$ & $65.9(8.9)^{*}$ \\
\hline Female & 55.2 & 55.1 & 55.1 & 55.4 & 55.3 \\
\hline Income $<\$ 20 \mathrm{k}$ & 15.2 & 16.7 & 15.3 & 15.3 & $13.8^{*}$ \\
\hline Education $<$ high school & 9.3 & 11.6 & 10.2 & 8.9 & $7.1^{*}$ \\
\hline Hypertension & 56.3 & 52.9 & 55.9 & 56.9 & $58.7^{*}$ \\
\hline Diabetes mellitus & 17.8 & 17.3 & 17.3 & 18.4 & 18.0 \\
\hline Waist circumference, $\mathrm{cm}$ & 57.8 & 56.9 & 58.3 & 57.7 & 58.4 \\
\hline OBS years & $12.7(3.7)$ & $7.6(1.4)$ & $11.0(0.81)$ & $14.0(0.82)$ & $17.5(1.5)^{*}$ \\
\hline
\end{tabular}

Values for age, body mass index, calories, eGFR, ACR and OBS years are reported as mean $( \pm S D)$. Values for race, sex, current smoking status, income, education, hypertension, diabetes and waist circumference are reported as percentage.

${ }^{*} \mathrm{p}<0.001$ based on the analysis of variance for continuous variables and based on chi-square test for categorical variables.

\section{Results}

There were 19,461 subjects included in the analyses. Their mean age (SD) was 64.8 (9.2) years, and $55.2 \%$ were women and $67.4 \%$ were white (table 2 ). The mean (SD) OBS was 12.7 (3.7), with median of 13 and interquartile range of 10-15. The proportion of white participants was lower in patients with lower versus those with higher OBS ( $\mathrm{Q} 1=66.3 \%$ vs. $\mathrm{Q} 4=69.6 \%)$.

Higher OBS quartiles (i.e., those with increasing predominance of antioxidants and lower pro-oxidants) included lower proportions of participants in the stroke belt and buckle region. Higher OBS quartiles were associated with lower likelihood of smoking, greater income and education level, and higher likelihood of self-reported hypertension and statin and diabetic medication use.

Table 1 (online suppl. table 1; for all online suppl. material, see www.karger.com/doi/10.1159/000441623) shows the distribution of the individual score components by OBS quartiles. NSAID use was higher in higher versus lower OBS quartiles. Aspirin use also higher in higher versus lower OBS quartiles. The prevalence of heavy alcohol use was lowest in Q4. The intake of antioxidants (vitamin $\mathrm{C}, \alpha$-carotene, $\beta$-carotene, vitamin $\mathrm{E}$, lutein, and lycopene and cryptoxanthin levels) was highest in Q4. However, pro-oxidant PUFAs and iron were also higher in Q4.

Table 3 shows the crude association between the OBS quartiles and the outcomes: albuminuria, CKD and ESRD. The crude ORs (95\% CI) were estimated for CKD and albuminuria, and the crude HRs (95\% CI) were estimated for ESRD. A total of 2,519 (12.9\%) had prevalent albuminuria, 1,957 participants had prevalent CKD (10.0\%) and $90(0.46 \%)$ participants developed ESRD over a median follow-up period of 3.5 years (range 2.144.32 years). In summary, the crude associations (OR and $\mathrm{HR}$ ) reflecting the association between OBS quartile and each of the individual outcomes was significant for macroalbuminuria ( $\mathrm{p}$ for trend $<0.01$ ) but not for albuminuria, CKD or ESRD (table 3). When analyzed as a continuous variable, in the crude model, for every 5-unit increase in the OBS, the odds of having albuminuria was 0.98 times the odds of not having albuminuria (OR 0.98, 95\% CI $0.93-1.04$ ) and the odds of having macroalbuminuria was 0.78 times the odds of no macroalbuminuria (OR 0.78, 95\% CI 0.68-0.90; table 4). The results for a 10unit increase in OBS are also shown in table 3. In the crude model, for every 5-unit increase in OBS, the odds 

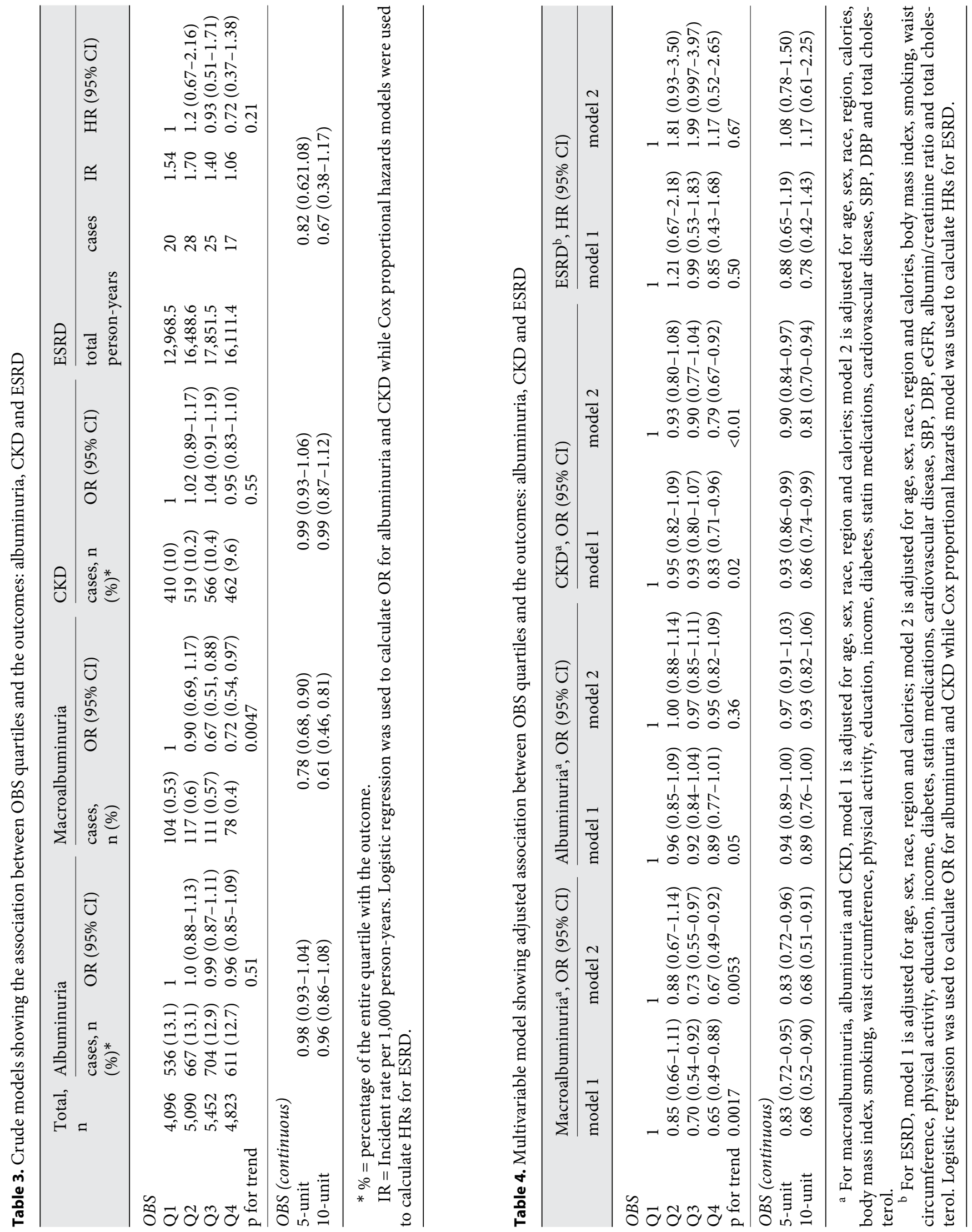
of CKD was 0.99 times the odds of not having CKD (OR $0.99,95 \%$ CI $0.93-1.06$ ). For every 5 -unit increase in OBS in the crude model, the likelihood of developing ESRD was 0.82 that of not developing ESRD (HR $0.75,95 \%$ CI $0.55-1.01)$. The crude associations between OBS as a continuous variable and the outcomes (albuminuria, CKD and ESRD) were not statistically significant.

Table 4 shows the associations of OBS with albuminuria, macroalbuminuria, CKD and ESRD, adjusted for various confounders. There was also no significant association between OBS quartiles and albuminuria in the fully adjusted model; however, for macroalbuminuria, compared to Q1, Q3 (OR 0.73, 95\% CI 0.55-0.97) and Q4 (OR $0.67,95 \%$ CI $0.49-0.92$ ) were associated with a lower odds of macroalbuminuria (table 4). The trend for macroalbuminuria was also significant for model 1 ( $\mathrm{p}$ trend $<0.01$ ) but not for model 2 ( $\mathrm{p}$ trend $=0.19$ ) OBS in the highest versus lowest quartile was associated with significantly lower odds of CKD (OR 0.83, 95\% CI 0.71-0.96) in model 1. The trend for OBS was also significant for CKD in model 1 ( $\mathrm{p}$ trend $=0.02$ ). This statistically significant inverse association remained after adjusting for other covariates in model 2 (OR 0.71, 95\% CI 0.60-0.831). Those in Q4 had a $29 \%$ lower odds of having an eGFR $<60 \mathrm{ml} /$ $\min / 1.73 \mathrm{~m}^{2}$. The trend test for OBS in model 2 was also statistically significant ( $\mathrm{p}$ trend $<0.0001$ ).

Using OBS as a continuous variable, for a 5-unit increase in OBS, the odds of having macroalbuminuria was 0.77 times the odds of no macroalbuminuria in model 1 but not significant in model 2. In the crude models, there was no association between the odds of having CKD and a 5-unit increase in OBS (table 3). However, in model 1, (table 4), the odds of having CKD versus no CKD for 5 -unit increase in OBS was 0.93 , and this was statistically significant (95\% CI 0.86-0.99). In the fully adjusted model, the odds of having CKD versus no CKD for a 5-unit increase in OBS was 0.90 (95\% CI 0.84-0.97). The association between OBS and ESRD was not statistically significant (tables 3 and 4).

A sensitivity analysis excluding NSAIDs and aspirin from the OBS showed that the association between the OBS and CKD was significant in the crude and adjusted models ( $\mathrm{p}$ for trend $<0.001 \mathrm{in}$ all the models). In addition, Q2 (OR 0.86, 95\% CI 0.75-1.00) and Q3 (OR 0.71, 95\% CI 0.60-0.83) were associated with significantly lower odds of having CKD. The results were also similar when OBS was analyzed as a continuous variable (online suppl. table 2).

OBS was significantly associated with albuminuria using linear regression models. A 5-unit increase in OBS was associated with a $7.30 \mathrm{mg} / \mathrm{g}$ reduction in albuminuria in the crude model $(\mathrm{p}<0.01)$, and a reduction of 6.91 $\mathrm{mg} / \mathrm{g}$ reduction in albuminuria $(\mathrm{p}<0.01)$. For a 5 -unit increase in OBS, the eGFR changes by $0.57 \mathrm{ml} / \mathrm{min} / 1.73$ $\mathrm{m}^{2}(\mathrm{p}<0.01)$ in the crude model and $0.88 \mathrm{ml} / \mathrm{min} / 1.73$ $\mathrm{m}^{2}$ in the fully adjusted model $(\mathrm{p}<0.001$; online suppl. table 3).

When we investigated the crude association between albuminuria and the individual pro- and antioxidant, we found unsaturated fats $(\mathrm{p}<0.01)$, iron $(\mathrm{p}<0.01)$, a-carotene $(\mathrm{p}<0.0001)$, lutein $(\mathrm{p}<0.01)$, NSAIDs $(\mathrm{p}<$ $0.05)$, smoking $(\mathrm{p}<0.0001)$, selenium $(\mathrm{p}<0.05)$, aspirin $(\mathrm{p}<0.0001)$ and alcohol $(\mathrm{p}<0.0001$; online suppl. table 4$)$.

The crude analysis of the association between pro and anti-oxidant factors and CKD showed that unsaturated fat $(\mathrm{p}<0.0001)$, iron $(\mathrm{p}<0.05)$, lycopene $(\mathrm{p}<0.01)$, $\alpha$-carotene $(\mathrm{p}<0.001), \beta$-carotene $(\mathrm{p}<0.0001)$, lutein $(\mathrm{p}<0.0001), \beta$-cryptoxanthin $(\mathrm{p}<0.01)$, $\alpha$-tocopherol $(\mathrm{p}<0.0001)$, smoking $(\mathrm{p}<0.001)$, aspirin $(\mathrm{p}<0.0001)$ and alcohol $(\mathrm{p}<0.001)$ were all individually associated with CKD (online suppl. table 4).

\section{Discussion}

We examined the association between OBS, albuminuria, CKD and ESRD and found that higher OBS quartiles (which reflect an increased antioxidant effect) were associated with lower prevalence of $\mathrm{CKD}$, as measured by eGFR $<60 \mathrm{ml} / \mathrm{min} / 1.73 \mathrm{~m}^{2}$ and associated with albuminuria in the linear regression models and macroalbuminuria in the crude and partially adjusted models but not associated incident ESRD.

Our study suggests the greater the shift from pro- to antioxidant exposures (as measured by higher OBS), the lower the odds of CKD as defined by an eGFR $<60 \mathrm{ml} /$ $\mathrm{min} / 1.73 \mathrm{~m}^{2}$. To our knowledge, this is the first study that utilizes the OBS to determine the association between the balance of pro- and antioxidant exposure and kidney disease.

The lack of association seen between OBS and incident ESRD are inconclusive due to the small number of participants who developed ESRD and the relatively short follow-up period. This warrants further investigation into the association between OBS and ESRD when there is longer follow-up data available. Other possible explanations of this are that since the FFQ is measured at baseline, secular dietary changes, particularly those that may occur among participants with prevalent CKD who have many dietary restrictions are not accounted for. It also 
suggests that the temporal association between OBS and renal damage remains unclear.

There was a significant inverse association between OBS and CKD as defined by eGFR $<60 \mathrm{ml} / \mathrm{min} / 1.73 \mathrm{~m}^{2}$ that became more pronounced as we adjusted for potential confounders. Current evidence suggests that CKD is a pro-oxidant state as evidenced by the increase in oxidative markers in atherosclerotic lesions of patients with CKD as well as in circulating plasma of CKD patients [37-39]. Our results suggest that the OBS may be a measure of pro- and antioxidant exposure in CKD. Measuring the OBS may be the first step in detecting pro- and antioxidant exposure in individuals at risk for development of an eGFR $<60 \mathrm{ml} / \mathrm{min} / 1.73 \mathrm{~m}^{2}$. These results need to be further validated using incident CKD cases in a dedicated CKD cohort.

OBS has been validated in previous studies. Kong et al. [40] validated the OBS in all-cause, cancer and non-cancer mortality in the REGARDS cohort. Dash et al. [21] also validated the OBS in a pooled case-control study of incident, sporadic colorectal adenoma. OBS was also found to be associated with circulating biomarkers of oxidative stress, F2-isoprostanes and C-reactive protein, in colorectal adenoma [41].

The strengths of the study include the use of a large population cohort. The OBS provides a measure whereby the effects of pro- and antioxidant factors in the diet and medications are summed up, and this 'effect' or 'balance' can be used to determine outcomes. The a priori selection of cutoffs in the components of OBS reduces subjectivity in measurement [12].

The limitations of the study include the small number of incident ESRD cases in the cohort and misclassification due to self-reported data, particularly dietary assessments from the food frequency questionnaire, to determine the OBS. Although dietary questionnaires are prone to recall bias and nutritional intake recorded may be inaccurate, on the population level, they provide a satisfactory classifica- tion of nutrient intake $[27,42,43]$. The study of CKD and albuminuria was cross-sectional with selection bias from missing data and residual confounding. The OBS was limited to dietary/lifestyle exposures and included no endogenous measures of antioxidant cell function $[44,45]$.

The goal of identifying the individuals at risk for higher oxidative stress is to institute aggressive risk modification. This study suggests that higher OBS may be independently associated with lower prevalence of CKD and may represent a modifiable risk factor for CKD.

\section{Acknowledgments}

This research project is supported by a cooperative agreement U01 NS041588 from the National Institute of Neurological Disorders and Stroke, National Institutes of Health, Department of Health and Human Service. The content is solely the responsibility of the authors and does not necessarily represent the official views of the National Institute of Neurological Disorders and Stroke or the National Institutes of Health. Representatives of the funding agency have been involved in the review of the manuscript but not directly involved in the collection, management, analysis or interpretation of the data. The authors thank other investigators, the staff and the participants of the REGARDS study for their valuable contributions. A full list of participating REGARDS investigators and institutions can be found at http://www. regardsstudy.org.

T.I. is in part funded by the PRIDE scholarship, R25 HL105401 National Heart, Lung, Blood and Sleep Institute, National Institutes of Health. This was presented as an abstract at the ASN meeting in Atlanta 2014.

We would like to thank Dr. Nosayaba Enofe for proof-reading the manuscript and thank Miss Xiao Wang for additional data analysis addressing the reviewers' comments.

\section{Disclosure Statement}

The results presented in this paper have not been published previously in whole or part, except in abstract format. The authors have no conflict of interest.

\section{References}

1 Bachem MG, Wendelin D, Schneiderhan W, Haug C, Zorn U, Gross HJ, Schmid-Kotsas A, Grünert A: Depending on their concentration oxidized low density lipoproteins stimulate extracellular matrix synthesis or induce apoptosis in human coronary artery smooth muscle cells. Clin Chem Lab Med 1999;37:319-326.

2 Galle J, Wanner C: Modification of lipoproteins in uremia: oxidation, glycation and carbamoylation. Miner Electrolyte Metab 1999; 25:263-268.
3 Singal PK, Kapur N, Dhillon KS, Beamish RE, Dhalla NS: Role of free radicals in catecholamine-induced cardiomyopathy. Can J Physiol Pharmacol 1982;60:1390-1397.

4 Dhalla AK, Hill MF, Singal PK: Role of oxidative stress in transition of hypertrophy to heart failure. J Am Coll Cardiol 1996;28:506-514.

5 Reuter S, Gupta SC, Chaturvedi MM, Aggarwal BB: Oxidative stress, inflammation, and cancer: how are they linked? Free Radic Biol Med 2010;49:1603-1616.
6 Tucker PS, Dalbo VJ, Han T, Kingsley MI Clinical and research markers of oxidative stress in chronic kidney disease. Biomarkers 2013;18:103-115.

7 Aveles PR, Criminácio CR, Gonçalves S, Bignelli AT, Claro LM, Siqueira SS, Nakao LS, PecoitsFilho R: Association between biomarkers of carbonyl stress with increased systemic inflammatory response in different stages of chronic kidney disease and after renal transplantation. Nephron Clin Pract 2010;116:c294-c299. 
8 Oberg BP, McMenamin E, Lucas FL, McMonagle E, Morrow J, Ikizler TA, Himmelfarb J: Increased prevalence of oxidant stress and inflammation in patients with moderate to severe chronic kidney disease. Kidney Int 2004;65:1009-1016.

9 L Gupta K, Sahni N: Dietary antioxidents and oxidative stress in predialysis chronic kidney disease patients. J Nephropathol 2012;1:134142.

10 Stenvinkel P, Holmberg I, Heimbürger O, Diczfalusy U: A study of plasmalogen as an index of oxidative stress in patients with chronic renal failure. Evidence of increased oxidative stress in malnourished patients. Nephrol Dial Transplant 1998;13:2594-2600.

11 Bruce WR, Giacca A, Medline A: Possible mechanisms relating diet and risk of colon cancer. Cancer Epidemiol Biomarkers Prev 2000;9:1271-1279.

12 Goodman M, Bostick RM, Dash C, Terry P, Flanders WD, Mandel J: A summary measure of pro- and anti-oxidant exposures and risk of incident, sporadic, colorectal adenomas. Cancer Causes Control 2008;19:1051-1064.

13 Goodman M, Bostick RM, Dash C, Flanders WD, Mandel JS: Hypothesis: oxidative stress score as a combined measure of pro-oxidant and antioxidant exposures. Ann Epidemiol 2007;17:394-399.

14 Wright ME, Mayne ST, Stolzenberg-Solomon RZ, Li Z, Pietinen P, Taylor PR, Virtamo J, Albanes D: Development of a comprehensive dietary antioxidant index and application to lung cancer risk in a cohort of male smokers. Am J Epidemiol 2004;160:68-76.

15 Van Hoydonck PG, Temme EH, Schouten EG: A dietary oxidative balance score of vitamin $\mathrm{C}$, beta-carotene and iron intakes and mortality risk in male smoking Belgians. J Nutr 2002;132:756-761.

16 Goodman M, Bostick RM, Gross M, Thyagarajan B, Dash C, Flanders WD: Combined measure of pro- and anti-oxidant exposures in relation to prostate cancer and colorectal adenoma risk: an update. Ann Epidemiol 2010;20:955-957.

17 Lynch KE, Lynch R, Curhan GC, Brunelli SM: Prescribed dietary phosphate restriction and survival among hemodialysis patients. Clin J Am Soc Nephrol 2011;6:620-629.

18 Mc Causland FR, Waikar SS, Brunelli SM: Increased dietary sodium is independently associated with greater mortality among prevalent hemodialysis patients. Kidney Int 2012; 82:204-211.

19 Gutiérrez OM, Muntner P, Rizk DV, McClellan WM, Warnock DG, Newby PK, Judd SE: Dietary patterns and risk of death and progression to ESRD in individuals with CKD: a cohort study. Am J Kidney Dis 2014;64:204-213.

20 Labadie J, Goodman M, Thyagarajan B, Gross M, Sun Y, Fedirko V, Bostick RM: Associations of oxidative balance-related exposures with incident, sporadic colorectal adenoma according to antioxidant enzyme genotypes. Ann Epidemiol 2013;23:223-226.

21 Dash C, Goodman M, Flanders WD, Mink PJ, McCullough ML, Bostick RM: Using pathway-specific comprehensive exposure scores in epidemiology: application to oxidative balance in a pooled case-control study of incident, sporadic colorectal adenomas. Am J Epidemiol 2013;178:610-624.

22 Slattery ML, Lundgreen A, Welbourn B, Wolff RK, Corcoran C: Oxidative balance and colon and rectal cancer: interaction of lifestyle factors and genes. Mutat Res 2012;734:30-40.

23 Howard VJ, Cushman M, Pulley L, Gomez CR, Go RC, Prineas RJ, Graham A, Moy CS, Howard G: The reasons for geographic and racial differences in stroke study: objectives and design. Neuroepidemiology 2005;25: 135-143.

24 Block G, Woods M, Potosky A, Clifford C: Validation of a self-administered diet history questionnaire using multiple diet records. J Clin Epidemiol 1990;43:1327-1335.

25 Mares-Perlman JA, Klein BE, Klein R, Ritter LL, Fisher MR, Freudenheim JL: A diet history questionnaire ranks nutrient intakes in middle-aged and older men and women similarly to multiple food records. J Nutr 1993; 123:489-501.

26 Caan BJ, Slattery ML, Potter J, Quesenberry CP Jr, Coates AO, Schaffer DM: Comparison of the Block and the Willett self-administered semiquantitative food frequency questionnaires with an interviewer-administered dietary history. Am J Epidemiol 1998;148: 1137-1147.

27 Judd SE, Aaron KJ, Letter AJ, Muntner P, Jenny NS, Campbell RC, Kabagambe EK, Levitan EB, Levine DA, Shikany JM, et al: High sodium: potassium intake ratio increases the risk for all-cause mortality: the Reasons for Geographic and Racial Differences in Stroke (REGARDS) study. J Nutr Sci 2013;2:e13.

28 United States Renal Data System: 2012 USRDS annual data report: Atlas of Chronic Kidney Disease and End-Stage Renal Disease in the United States; Bethesda, National Institutes of Health, National Institute of Diabetes and Digestive and Kidney Diseases, 2012.

29 Lash JP, Go AS, Appel LJ, He J, Ojo A, Rahman M, Townsend RR, Xie D, Cifelli D, Cohan J, et al: Chronic renal insufficiency cohort (CRIC) study: baseline characteristics and associations with kidney function. Clin J Am Soc Nephrol 2009;4:1302-1311.

30 Orth SR, Stöckmann A, Conradt C, Ritz E, Ferro M, Kreusser W, Piccoli G, Rambausek M, Roccatello D, Schäfer K, et al: Smoking as a risk factor for end-stage renal failure in men with primary renal disease. Kidney Int 1998; 54:926-931

31 Stengel B, Couchoud C, Cénée S, Hémon D: Age, blood pressure and smoking effects on chronic renal failure in primary glomerular nephropathies. Kidney Int 2000;57:2519-2526.
32 Shankar A, Klein R, Klein BE: The association among smoking, heavy drinking, and chronic kidney disease. Am J Epidemiol 2006;164: 263-271.

33 Hallan SI, Orth SR: Smoking is a risk factor in the progression to kidney failure. Kidney Int 2011;80:516-523.

34 Kronborg J, Solbu M, Njølstad I, Toft I, Eriksen BO, Jenssen T: Predictors of change in estimated GFR: a population-based 7-year follow-up from the Tromso study. Nephrol Dial Transplant 2008;23:2818-2826.

35 Newby PK, Noel SE, Grant R, Judd S, Shikany JM, Ard J: Race and region have independent and synergistic effects on dietary intakes in black and white women. Nutr J 2012;11:25.

36 Levey AS, Stevens LA, Schmid CH, Zhang YL, Castro AF 3rd, Feldman HI, Kusek JW, Eggers $\mathrm{P}$, Van Lente F, Greene T, et al: A new equation to estimate glomerular filtration rate. Ann Intern Med 2009;150:604-612.

37 Malle E, Woenckhaus C, Waeg G, Esterbauer $\mathrm{H}$, Gröne EF, Gröne HJ: Immunological evidence for hypochlorite-modified proteins in human kidney. Am J Pathol 1997;150:603615.

38 Sakata N, Imanaga Y, Meng J, Tachikawa Y, Takebayashi S, Nagai R, Horiuchi S: Increased advanced glycation end products in atherosclerotic lesions of patients with endstage renal disease. Atherosclerosis 1999;142: 67-77.

39 Satoh M, Yamasaki Y, Nagake Y, Kasahara J, Hashimoto M, Nakanishi N, Makino H: Oxidative stress is reduced by the long-term use of vitamin E-coated dialysis filters. Kidney Int 2001;59:1943-1950.

40 Kong SY, Goodman M, Judd S, Bostick RM, Flanders WD, McClellan W: Oxidative balance score as predictor of all-cause, cancer, and noncancer mortality in a biracial US cohort. Ann Epidemiol 2015;25:256.e1-262.e1.

41 Kong SY, Bostick RM, Flanders WD, McClellan WM, Thyagarajan B, Gross MD, Judd S, Goodman M: Oxidative balance score, colorectal adenoma, and markers of oxidative stress and inflammation. Cancer Epidemiol Biomarkers Prev 2014;23:545-554.

42 Dyer A, Elliott P, Chee D, Stamler J: Urinary biochemical markers of dietary intake in the INTERSALT study. Am J Clin Nutr 1997;65(4 suppl):1246S-1253S.

43 Toft U, Kristoffersen L, Ladelund S, Bysted A, Jakobsen J, Lau C, Jørgensen T, Borch-Johnsen K, Ovesen L: Relative validity of a food frequency questionnaire used in the Inter 99 study. Eur J Clin Nutr 2008;62:1038-1046.

44 Thomas SR, Neuzil J, Mohr D, Stocker R: Coantioxidants make alpha-tocopherol an efficient antioxidant for low-density lipoprotein. Am J Clin Nutr 1995;62(6 suppl):1357S$1364 \mathrm{~S}$.

45 Klaunig JE, Kamendulis LM: The role of oxidative stress in carcinogenesis. Annu Rev Pharmacol Toxicol 2004;44:239-267. 